\title{
DUBLIN RADIOCARBON DATES III
}

P Q DRESSER*

Physical Laboratory, University of Dublin, Dublin 2, Ireland

\section{INTRODUCTION}

The dating equipment and operating procedures in the Physical laboratory remained as described previously ( $R, 1974, \mathrm{v} 16$, no. 1, p 6-9).

Dates were calculated using the 5568-year half-life with 1950 as the reference year, the modern standard being 0.95 of the activity of the NBS oxalic acid standard. When done, $\delta^{13} \mathrm{C}$ was measured on $\mathrm{CO}_{2}$ produced in the combustion of samples, and results are given relative to the PDB standard. All samples were from Ireland.

\section{ACKNOWLEDGMENTS}

I am very grateful to $\mathrm{R} L$ Otlet of the Harwell laboratory, who measured the ${ }^{14} \mathrm{C}$ activity and $\delta^{13} \mathrm{C}$ ratios of some of the samples and accepted samples of benzene prepared in this laboratory. He kindly allowed me to publish the results in this list; the relevant samples are indicated in the text. Thanks are also due to $\mathrm{T} J$ Fitzgerald for making the remaining $\delta^{13} \mathrm{C}$ measurements. Financial support from the National Science Council of Ireland is gratefully acknowledged.

\section{ARCHAEOLOGIC SAMPLES}

\section{Rathgall series, Co Wicklow}

Charcoal samples are from a multivallate hillfort with extensive Early Bronze Age settlement and later occupations (Orpen, 1911; Raftery, 1970; 1971) at Rathgall, Rath East townland, 5km E of Tullow, Co Carlow (52 $48^{\prime} \mathrm{N}, 7^{\circ} 40^{\prime} \mathrm{W}$, NGR: S 902731) alt 122m. Coll and subm 1969 and 1970 by B Raftery, Dept Archaeol, Univ College, Dublin.

\section{D-133. S151}

Charcoal from pit at SW corner of Hearth 2, Square 32. Comment (BR): late Bronze Age provenience.

\section{D-134. 5113}

$2860 \pm 110$

Charcoal from Hearth 1, Square 25. Comment (BR): sample from initial late Bronze Age occupation.

D-135. S140

Charcoal. Wood and cereal grain from upper levels of ditch surrounding circular house, Square 31 .

\section{D-132. $\mathbf{S 4 0 0}$}

$$
\begin{array}{r}
2960 \pm 110 \\
\delta^{13} C=-25.8 \% \text { o }
\end{array}
$$

Charcoal from upper levels of large basket-lined storage pit outside circular house, Square 18.

* Present address: Radiocarbon Dating Laboratory, Department of Plant Science, University College, Cardiff, CF1 1XL, Wales 
II. GEOLOGIC SAMPLES

\section{D-119. Fethard-on-sea, Co Wexford}

$$
\begin{array}{r}
4030 \pm 120 \\
\delta^{13} C=-26.7 \% \text { o }
\end{array}
$$

Tree roots in gray wedges in glacial deposit lying between high and low water marks on beach at Wood Village, Fethard-on-sea, $10.5 \mathrm{~km}$ ESE of Waterford $\left(52^{\circ} 12^{\prime} \mathrm{N}, 6^{\circ} 50^{\prime} \mathrm{W}\right.$, NGR: S 803035). Coll and subm 1972 by G F Mitchell, Dept Geol, Univ Dublin. Comment (GFM): possible explanation for date is that roots are last vestige of eroded submerged forest that grew at time indicated. Tree roots could not have penetrated to present location if sea stood at present level or higher.

\section{D-136F. Old Head, Co Mayo}

$$
\begin{array}{r}
10,010 \pm \mathbf{1 7 0} \\
\delta^{13} C=-25.8 \% \text { o }
\end{array}
$$

Fine insoluble material $<300 \mu \mathrm{m}$, from organic deposit $3 \mathrm{~cm}$ thick under $2 \mathrm{~m}$ glacial till exposed on beach at Old Head, $17 \mathrm{~km} \mathrm{~W}$ of Westport, Co Mayo $\left(53^{\circ} 47^{\prime} \mathrm{N}, 9^{\circ} 47^{\prime} \mathrm{W}\right.$, NGR: L 834823). Coll and subm 1972 by G F Mitchell. Comment: humic acid fraction dated; D-136H, $10,530 \pm 120$. Both samples pretreated and converted to benzene in Dublin lab, and counted in Harwell lab as HAR-627 (D-136F) and HAR-628 (D-136H).

\section{Dunmore Cave series, Co Kilkenny}

Samples coll during geologic and archaeologic investigations of cave system at Dunmore Cave, $9 \mathrm{~km} \mathrm{~N}$ of Kilkenny, Co Kilkenny $\left(52^{\circ} 44^{\prime} \mathrm{N}\right.$, $7^{\circ}$ 15' W, NGR: S 508658). Coll and subm 1973 by D Huddart and D Drew, Dept Geog, Dublin Univ. Comment: all results expresed as \% of modern and are uncorrected for isotopic fractionation.

\section{D-137. Sample 1A}

$$
40.2 \pm 0.9 \%
$$$$
\delta^{13} \mathrm{C}=-6.5 \% \text { o }
$$

Carbonate from upper $1 \mathrm{~cm}$ layer of flow-stone floor deposit, $10 \mathrm{~cm}$ thick, $3 \mathrm{~m}$ from excavated trench in rabbit burrow area.

\section{D-139. Sample 2}

$85.8 \pm 1.2 \%$

Charcoal from immediately above flow-stone floor deposit in excavated trench in rabbit burrow area. Comment: sample assoc with archaeologic deposit. Conventional age, $1230 \pm 110$ BP.

D-141. Sample 4A

$$
\begin{aligned}
& \mathbf{5 3 . 5} \pm \mathbf{0 . 9} \% \\
& \delta^{13} C=-6.0 \%
\end{aligned}
$$

Carbonate from central $2 \mathrm{~cm}$ core of pendant concretion, $12 \mathrm{~cm}$ diam, at cave entrance.

\section{D-142. Sample 4B}

$72.8 \pm 1.1 \%$ $\delta^{13} C=-0.5 \%$ D-141.

Carbonate from outer $2 \mathrm{~cm}$ layer of same concretion as Sample $4 \mathrm{~A}$, 


\section{D-144. Sample 5}

$$
\begin{array}{r}
\mathbf{0 . 7} \pm \mathbf{0 . 2 \%} \\
\delta^{1 s} C=-10.8 \%
\end{array}
$$

Carbonate from bottom $1 \mathrm{~cm}$ layer of flow-stone floor deposit, $5 \mathrm{~cm}$ thick, in Market Cross chamber. Comment: sample activity and $\delta^{13} \mathrm{C}$ measured at Harwell lab; HAR-634. Infinite age.

\section{D-149. Sample 9}

$$
\begin{aligned}
& 44.9 \pm 0.4 \% \\
& \delta^{13} C=-3.1 \%
\end{aligned}
$$

Carbonate from flow-stone floor deposit in Market Cross chamber. Comment: sample activity and $\delta^{13} \mathrm{C}$ measured in Harwell lab; HAR-637. Sample measured to determine relationship with Sample 5, D-144.

\section{D-147. Sample 7}

$114.1 \pm 2.6 \%$ $\delta^{13} C=-14.5 \%$

Drip-water sample coll Nov 1973 from Market Cross chamber. Comment: sample activity and $\delta^{13} \mathrm{C}$ measured in Harwell lab; HAR-635. Bicarbonate in drip-water precipitated as barium carbonate.

\section{D-148. Sample 8}

$142.6 \pm 1.4 \%$ $\delta^{13} \mathrm{C}=-26.4 \%$

Twigs grown summer 1973 from tree growing near cave entrance. Coll Nov 1973. Comment: sample activity $\delta^{13} C$ measured at Harwell lab; HAR-636.

\section{REFERENCES}

Orpen, G H, 1911, Rathgall, County Wicklow: Royal Soc Antiquaries Ireland Jour, $\mathrm{v} 41, \mathrm{p} 138-150$

Raftery, B, 1970, The Rathgall Hillfort, County Wicklow: Antiquity, v 44, p 51-54. p 296-298. 